\title{
A Multi-Dimensional Hausdorff Moment Problem: Regularization by Finite Moments
}

\author{
D. D. Ang, R. Gorenflo and D. D. Trong
}

Dedicated to Prof. Dr. G. Anger on occasion of his 70th birthday

\begin{abstract}
We consider the multi-dimensional Hausdorff moment problem over the unit cube: to reconstruct an unknown function from the (inaccurately) given values of the integrals of the unknown function multiplied by all power-products of the independent variables. We describe a regularization scheme using orthogonalization by the tensor product of (shifted) Legendre polynomials and "approximation" of the unknown function by a finite sum, the dimension of the space of approximation playing the role of the regularization parameter. For the case of square integrability of the unknown function we present an estimate of the regularization error that implies convergence if the data error tends to zero.
\end{abstract}

Keywords: Ill-posed problems, Hausdorff moment problem, polynomial approximation AMS subject classification: Primary 65 R 30, secondary 41 A 10

\section{Introduction}

We propose to present a regularization of a Hausdorff moment problem, with error estimates given. The literature on the moment problem is impressive, we shall list only a few of them in the bibliography (cf. $[2-8,11,13])$. Of particular relevance to the present work are the papers by Ang, Vy and Gorenflo [3] and by Talenti [11] (see also Inglese [7]). In [11], the author considered the one-dimensional case and proved the stability of the finite moment approximation with stability estimates given. But the relationship of the finite moment approximation to the original moment problem was not exhibited as transparently as we feel desirable. In the present paper, we shall consider the multi-dimensional case, specifically the following problem.

Problem (MP). Find a function $u$ in $L^{2}(I)$ satisfying the sequence of equations

$$
\int_{I} u\left(x_{1}, \ldots, x_{d}\right) x_{1}^{k_{1}} \cdots x_{d}^{k_{d}} d x_{1} \cdots d x_{d}=\mu_{k_{1} \cdots k_{d}} \quad\left(k_{i} \in \mathbb{N}_{0} ; i=1, \ldots, d\right)
$$

where $d \in \mathbb{N}, I=[0,1]^{d} \subset \mathbb{R}^{d}$ and $\mu=\left(\mu_{k_{1} \ldots k_{d}}\right)$ is a given bounded sequence of real numbers.

D. D. Ang: Nat. Univ., Dept. Math., 227 Nguyen Van Cu, Q5, Hochiminh City, Vietnam

R. Gorenflo: Freie Universität Berlin; FB Math. und Inf., Arnimallee 2-6, D-14195 Berlin

D. D. Trong: Nat. Univ., Dept. Math., 227 Nguyen Van Cu, Q5, Hochiminh City, Vietnam

ISSN 0232-2064 / \$2.50 C Heldermann Verlag Berlin 
It can be proved that (MP) is an ill-posed problem. Note also that (MP) does not always have a solution for an arbitrary bounded sequence $\left(\mu_{k_{1} \ldots k_{d}}\right)$ in the right-hand side of (1). Hence it is realistic to deal also with cases where problem (MP) does not have a solution. We refer to the paper [3] of which the present work can be seen as a continuation. However while [3] deals with a general moment problem, the Hausdorff moment problem has it own peculiarities that we have taken into account, in departure from the general case, especially in the crucial choice of the function $f(t),\left[f^{-1}\left(\varepsilon^{\frac{1}{2}}\right)\right]$ being the dimension of our finite approximation.

In our construction of finite-dimensional approximations, we obtain an orthonormalization of the basis functions $\left\{x_{1}^{k_{1}} \cdots x_{d}^{k_{d}}\right\} \quad\left(k_{i} \in \mathbb{N}_{0} ; i=1, \ldots, d\right)$ not through the Gram-Schmidt process but by using the polynomials

$$
L_{k_{1} \ldots k_{d}}\left(x_{1}, \ldots, x_{d}\right)=L_{k_{1}}\left(x_{1}\right) \cdots L_{k_{d}}\left(x_{d}\right)
$$

where

$$
L_{m}(x)=\sum_{j=0}^{m} C_{m j} x^{j} \quad\left(m \in \mathbb{N}_{0}\right)
$$

with

$$
C_{m j}=(2 m+1)^{\frac{1}{2}}(-1)^{j} \frac{(m+j) !}{(j !)^{2}(m-j) !} \quad(j=0,1, \ldots, m) .
$$

The sequence $\left(L_{k_{1}} \cdots L_{k_{d}}\right) \quad\left(k_{i} \in \mathbb{N}_{0} ; i=1, \ldots, d\right)$ forms a complete orthonormal set in $L^{2}(I)$. In fact,

$$
L_{m}(x)=(2 m+1)^{\frac{1}{2}} P_{m}(1-2 x),
$$

where $P_{m}$ is the Legendre polynomial:

$$
P_{m}(t)=\sum_{j=0}^{m} \frac{(m+j) !}{(j !)^{2}(m-j) ! 2^{j}}(t-1)^{j}, \quad\left\|P_{m}\right\|_{L^{2}(-1,1)}^{2}=\frac{2}{2 m+1}
$$

(cf. [10: page 227]). Thus $\left(L_{m}\right)$ is a complete orthonormal basis in $L^{2}(0,1)$ and hence $\left(L_{k_{1}} \cdots L_{k_{d}}\right)$ is a complete orthonormal set in $L^{2}(I)$. From (3), we have

$$
L_{k_{1} \cdots k_{d}}\left(x_{1}, \ldots, x_{d}\right)=\sum_{p_{1}=0}^{k_{1}} \cdots \sum_{p_{d}=0}^{k_{d}} C_{k_{1} p_{1}} \cdots C_{k_{d} p_{d}} x_{1}^{p_{1}} \cdots x_{d}^{p_{d}} .
$$
follows:

If $\mu=\left(\mu_{k_{1} \cdots k_{d}}\right)$ is a real sequence, we define the sequence $\lambda=\lambda(\mu)=\left(\lambda_{k_{1} \cdots k_{d}}\right)$ as

$$
\lambda_{k_{1} \cdots k_{d}}=\lambda_{k_{1} \cdots k_{d}}(\mu)=\sum_{p_{1}=0}^{k_{1}} \cdots \sum_{p_{d}=0}^{k_{d}} C_{k_{1} p_{1}} \cdots C_{k_{d} p_{d}} \mu_{p_{1} \cdots p_{d}} .
$$

Now put

$$
p^{n}=p^{n}(\mu)=\sum_{k_{1}, \ldots, k_{d}=0}^{n} \lambda_{k_{1} \cdots k_{d}}(\mu) L_{k_{1} \ldots k_{d}}
$$




\section{Results}

We now give our results in form of three theorems that we shall prove in Section 3.

Theorem 1. Let $\mu=\left(\mu_{k_{1}} \ldots k_{d}\right) \quad\left(k_{i} \in \mathbb{N}_{0} ; i=1, \ldots, d\right)$ be a given sequence of real numbers. If $u$ is the (unique) solution of (1) in $L^{2}(I)$, then

$$
\sum_{k_{1}, \ldots k_{d}=0}^{\infty}\left(\sum_{p_{1}, \ldots, p_{d}=0}^{\infty} C_{k_{1} p_{1}} \cdots C_{k_{d} p_{d}} \mu_{p_{1}} \cdots p_{d}\right)^{2}<\infty
$$

and

$$
p^{n}(\mu) \rightarrow u \quad \text { in } L^{2}(I) \text { as } n \rightarrow \infty,
$$

where $C_{i j}$ is defined by (4) if $j \leq i$ and $C_{i j}=0$ if $j>i$. Moreover, if the solution $u$ is in $H^{1}(I)$, then

$$
\left\|p^{n}(\mu)-u\right\| \leq \frac{1}{n+1}(F(u))^{\frac{1}{2}} \quad(n \in \mathbb{N})
$$

where $\|\cdot\|$ is the $L^{2}(I)$-norm and

$$
F(u)=\sum_{i=1}^{d} \int_{I} x_{i}\left(1-x_{i}\right)\left|\frac{\partial u}{\partial x_{i}}\right|^{2} d x_{1} \cdots d x_{d} .
$$

In Theorem 1 above, it is assumed that a solution exists. In the general case that a solution may not exist, Theorem 2 below will be useful.

Theorem 2. Let $u_{0} \in L^{2}(I)$ be the unique solution of (1) corresponding to $\mu^{0}=$ $\left(\mu_{k_{1}}^{0} \ldots k_{d}\right)\left(k_{i} \in \mathbb{N}_{0} ; i=1, \ldots, d\right)$ in the right-hand side of $(1)$. Let

$$
f(t)=\frac{1}{\sqrt{(2 \pi)^{d}}}(3+2 \sqrt{2})^{d t+d}
$$

and for $0<\epsilon<1$, put

$$
n(\varepsilon)=\left[f^{-1}\left(\varepsilon^{-\frac{1}{2}}\right)\right]
$$

where $[x]$ is the largest integer $n \leq x$. Then there exists a function $\eta(\varepsilon)(0<\varepsilon<1)$ such that $\eta(\varepsilon) \rightarrow 0$ as $\varepsilon \rightarrow 0$ and that for all sequences $\mu$ satisfying

$$
\left\|\mu-\mu^{0}\right\|_{\infty}:=\sup _{k_{1}, \ldots, k_{d} \in \mathbf{N}_{0}}\left|\mu_{k_{1} \ldots k_{d}}-\mu_{k_{1} \ldots k_{d}}^{0}\right| \leq \varepsilon
$$

we have $\left\|p^{n(\varepsilon)}(\mu)-u_{0}\right\| \leq \eta(\varepsilon)$. Moreover, if $u_{0} \in H^{1}(I)$ and $0<\varepsilon<\left(\frac{\sqrt{2 \pi}}{3+2 \sqrt{2}}\right)^{2 d}$, then

$$
\left\|p^{n(e)}(\mu)-u_{0}\right\| \leq \varepsilon^{\frac{1}{2}}+\frac{\left|F\left(u_{0}\right)\right|^{\frac{1}{2}}}{C_{1}+C_{2} \ln \left(\frac{1}{\varepsilon}\right)}
$$

where

$$
C_{1}=\frac{\ln (\sqrt{2 \pi}(3-2 \sqrt{2}))}{\ln (3+2 \sqrt{2})} \quad \text { and } \quad C_{2}=\frac{1}{2 d \ln (3+2 \sqrt{2})}
$$


Remark. In the first version of this paper (see also [2]), we had derived (13) with $C_{1}$ and $C_{2}$ replaced by

$$
\widetilde{C}_{1}=\frac{2 \ln \left(\frac{8 \sqrt{5}}{27 \sqrt{2}}\right)}{2 \ln 3+\frac{1}{2} \ln 2} \quad \text { and } \quad \widetilde{C}_{2}=\frac{1}{d\left(2 \ln 3+\frac{1}{2} \ln 2\right)}
$$

respectively.

Estimate (13) with $C_{1}$ and $C_{2}$ given by (14) was derived using formula (31) as suggested by one of the referees. Clearly, the constants $C_{1}$ and $C_{2}$ give a better approximation than do $\widetilde{C}_{1}$ and $\widetilde{C}_{2}$, respectively. While it is not known to us whether $C_{1}$ and $C_{2}$ are the best constants, we shall show below that the estimate of the order of magnitude of the error for $\varepsilon \rightarrow 0$ as given by (13) is optimal in the sense of the following theorem.

Theorem 3. Assume that there exist two functions $m:(0,1] \rightarrow \mathbb{N}$ and $\alpha:(0,1] \rightarrow$ $(0, \infty)$ such that $\lim _{\varepsilon \downarrow 0} m(\varepsilon)=+\infty$ and $\lim _{\varepsilon \downarrow 0} \alpha(\varepsilon)=0$, and

$$
\left\|p^{m(\varepsilon)}(\mu)-u_{0}\right\| \leq\left(1+\left(F\left(u_{0}\right)\right)^{\frac{1}{2}}\right) \alpha(\varepsilon)
$$

for all $u_{0} \in H^{1}(I), I=[0,1]^{d}$, and all $\mu=\left(\mu_{k_{1} \ldots k_{d}}\right)$ satisfying

$$
\left\|\mu-\mu_{0}\right\|_{\infty}=\sup _{k_{1}, \ldots, k_{d} \geq 0}\left|\mu_{k_{1} \ldots k_{d}}-\mu_{k_{1} \ldots k_{d}}^{0}\right| \leq \varepsilon,
$$

where

Then we have

$$
\mu_{k_{1} \ldots k_{d}}^{0}=\int_{l} u_{0}\left(x_{1}, \ldots, x_{d}\right) x_{1}^{k_{1}} \cdots x_{d}^{k_{d}} d x_{1} \cdots d x_{d}
$$

$$
\liminf _{\varepsilon 10} \alpha(\varepsilon) \ln \left(\frac{1}{\varepsilon}\right) \geq \frac{d}{2} \ln (3+2 \sqrt{2})
$$

\section{Proofs}

For definiteness, we shall give the proofs of Theorems $1-3$ in the case $d=3$ only. The proofs carry almost verbatim to the general case. For $d=3$, we put $\left(x_{1}, x_{2}, x_{3}\right)=$ $(x, y, z),\left(k_{1}, k_{2}, k_{3}\right)=(i, j, k), \mu_{k_{1} k_{2} k_{3}}=\mu_{i j k}, \lambda_{k_{1} k_{2} k_{3}}=\lambda_{i j k}$ and $L_{k_{1} k_{2} k_{3}}(x, y, z)=$ $L_{i}(x) L_{j}(y) L_{k}(z)=L_{i j k}(x, y, z)$.

Proof of Theorem 1. We easily get the first result of Theorem 1 using the orthonormality property of the $L_{i j k}$ 's. In fact, one has in view of (3)

$$
\begin{aligned}
\int_{I} u L_{i j k} d x d y d z & =\int_{I} u(x, y, z) \sum_{p=0}^{i} \sum_{q=0}^{j} \sum_{r=0}^{k} C_{i p} C_{j q} C_{k r} x^{p} y^{q} z^{r} d x d y d z \\
& =\sum_{p=0}^{i} \sum_{q=0}^{j} \sum_{r=0}^{k} C_{i p} C_{j q} C_{k r}\left(\int_{I} u(x, y, z) x^{p} y^{q} z^{r} d x d y d z\right) \\
& =\sum_{p=0}^{i} \sum_{q=0}^{j} \sum_{r=0}^{k} C_{i p} C_{j q} C_{k r} \mu_{i j k} \\
& =\lambda_{i j k} .
\end{aligned}
$$


Hence, by completeness and the orthonormality property of the $L_{i j k}$ 's in $L^{2}(I)$ one has

$$
u=\sum_{i, j, k=0}^{\infty} \lambda_{i j k}(\mu) L_{i j k} \quad \text { and } \quad\|u\|^{2}=\sum_{i, j, k=0}^{\infty}\left|\lambda_{i j k}(\mu)\right|^{2}<\infty,
$$

i.e. (8) holds. To prove (9), we subtract the first equation in (17) from (7) to get

$$
p^{n}(\mu)-u=-\sum_{m a x(i, j, k)>n} \lambda_{i j k}(\mu) L_{i j k} .
$$

Hence

$$
\left\|p^{n}(\mu)-u\right\|^{2}=\sum_{\max (i, j, k)>n}\left|\lambda_{i j k}(\mu)\right|^{2} .
$$

Combining (8) and (18) gives (9).

For the proof of (10) we rely, with $\alpha_{k}=\int_{0}^{1} v(x) L_{k}(x) d x$, on the identity (cf. [11])

$$
\int_{0}^{1} x(1-x)\left|v^{\prime}(x)\right|^{2} d x=\sum_{k=0}^{\infty} k(k+1) \alpha_{k}^{2} \quad \forall v \in H^{1}(0,1) .
$$

In view of (17) and (19), we have

$$
\begin{aligned}
& \sum_{i, j, k=1}^{\infty} i^{2} \lambda_{i j k}^{2} \leq \int_{I} x(1-x)\left|\frac{\partial u}{\partial x}(x, y, z)\right|^{2} d x d y d z \\
& \sum_{i, j, k=1}^{\infty} j^{2} \lambda_{i j k}^{2} \leq \int_{I} y(1-y)\left|\frac{\partial u}{\partial y}(x, y, z)\right|^{2} d x d y d z \\
& \sum_{i, j, k=1}^{\infty} k^{2} \lambda_{i j k}^{2} \leq \int_{I} z(1-z)\left|\frac{\partial u}{\partial z}(x, y, z)\right|^{2} d x d y d z .
\end{aligned}
$$

Adding (20) - (22) together, we get

$$
\sum_{i, j, k=1}^{\infty}\left(i^{2}+j^{2}+k^{2}\right) \lambda_{i j k}^{2} \leq F(u)
$$

where, we recall,

$$
\begin{aligned}
F(u)= & \int_{I}\left(x(1-x)\left|\frac{\partial u}{\partial x}(x, y, z)\right|^{2}+y(1-y)\left|\frac{\partial u}{\partial y}(x, y, z)\right|^{2}\right. \\
& \left.+z(1-z)\left|\frac{\partial u}{\partial z}(x, y, z)\right|^{2}\right) d x d y d z
\end{aligned}
$$

Note that

$$
i^{2}+j^{2}+k^{2} \geq(n+1)^{2} \quad \text { for } \max (i, j, k)>n .
$$

We get in view of (18), (23) and (24) that

$$
(n+1)^{2}\left\|p^{n}(\mu)-u\right\|^{2} \leq \sum_{\max (i, j, k)>n}\left(i^{2}+j^{2}+k^{2}\right) \lambda_{i j k}^{2}(\mu) \leq F(u),
$$

which gives the desired inequality. The proof of Theorem 1 is completed 
Proof of Theorem 2. We have

$$
\left\|p^{n}(\mu)-u_{0}\right\| \leq\left\|p^{n}(\mu)-p^{n}\left(\mu^{0}\right)\right\|+\left\|p^{n}\left(\mu^{0}\right)-u_{0}\right\| \quad(n \in \mathbb{N}) .
$$

We shall estimate the two terms in the right-hand side of (25). Using (18) gives

$$
\left\|p^{n}\left(\mu^{0}\right)-u_{0}\right\|^{2}=\sum_{\max (i, j, k)>n}\left(\sum_{p, q, r=0}^{\infty} C_{i p} C_{j q} C_{k r} \mu_{p q r}^{0}\right)^{2} .
$$

For the first term, we get in view of (7)

$$
p^{n}(\mu)-p^{n}\left(\mu^{0}\right)=\sum_{i, j, k=0}^{n} \sum_{p, q, r=0}^{\infty} C_{i p} C_{j q} C_{k r}\left(\mu_{p q r}-\mu_{p q r}^{0}\right) L_{i j k} .
$$

Hence

$$
\begin{aligned}
\left\|p^{n}(\mu)-p^{n}\left(\mu^{0}\right)\right\|^{2} & =\sum_{i, j, k=0}^{n}\left(\sum_{p, q, r=0}^{\infty} C_{i p} C_{j q} C_{k r}\left(\mu_{p q r}-\mu_{p q r}^{0}\right)\right)^{2} \\
& \leq\left\|\mu-\mu^{0}\right\|_{\infty}^{2} \sum_{i, j, k=0}^{n}\left(\sum_{p, q, r=0}^{\infty}\left|C_{i p} C_{j q} C_{k r}\right|\right)^{2}
\end{aligned}
$$

Noting that $C_{m j}=0$ for $j>m$, we have

$$
\sum_{p, q, r=0}^{\infty}\left|C_{i p} C_{j q} C_{k r}\right|=\sum_{p=0^{*}}^{i}\left|C_{i p}\right| \sum_{q=0}^{j}\left|C_{j q}\right| \sum_{r=0}^{k}\left|C_{k r}\right| .
$$

Substituting (29) into (28) gives

$$
\left\|p^{n}(\mu)-p^{n}\left(\mu^{0}\right)\right\|^{2} \leq\left\|\mu-\mu^{0}\right\|_{\infty}^{2}\left(\sum_{i=0}^{n}\left(\sum_{p=0}^{i}\left|C_{i p}\right|\right)^{2}\right)^{3} .
$$

From (3) - (5), one gets

$$
\sum_{p=0}^{i}\left|C_{i p}\right|=L_{i}(-1)=(2 i+1)^{\frac{1}{2}} P_{i}(3)
$$

On the other hand, one has (cf. [9: page 48], or [1: Equation (22.10.10)])

$$
P_{i}(3)=\frac{1}{\pi} \int_{0}^{\pi}(3+2 \sqrt{2} \cos \phi)^{i} d \phi \quad\left(i \in \mathbb{N}_{0}\right)
$$

We claim that

$$
P_{i}(3) \leq 2 \sqrt{\frac{3+2 \sqrt{2}}{\pi \sqrt{2}}} \cdot \frac{1}{\sqrt{2 i+1}}(3+2 \sqrt{2})^{i} \quad\left(i \in \mathbb{N}_{0}\right)
$$


the proof of which will be given later. Using (31) and (32) one gets after some rearrangements

$$
\sum_{p=0}^{i}\left|C_{i p}\right| \leq 2 \sqrt{\frac{3+2 \sqrt{2}}{\pi \sqrt{2}}}(3+2 \sqrt{2})^{i} \quad\left(i \in \mathbb{N}_{0}\right)
$$

and

$$
\sum_{i=0}^{n}\left(\sum_{p=0}^{i}\left|C_{i p}\right|\right)^{2} \leq \frac{4(3+2 \sqrt{2})}{\pi \sqrt{2}} \sum_{i=0}^{n}(3+2 \sqrt{2})^{2 i} \leq \frac{1}{2 \pi}(3+2 \sqrt{2})^{2 n+2} .
$$

Substituting the latter inequality into $(30)$ gives

$$
\left\|p^{n}(\mu)-p^{n}\left(\mu^{0}\right)\right\| \leq\left\|\mu-\mu^{0}\right\|_{\infty} \frac{1}{\sqrt{8 \pi^{3}}}(3+2 \sqrt{2})^{3 n+3} .
$$

Put

$$
f(t)=\frac{1}{\sqrt{8 \pi^{3}}}(3+2 \sqrt{2})^{3 t+3} \quad \text { and } \quad n(\varepsilon)=\left[f^{-1}\left(\varepsilon^{-\frac{1}{2}}\right)\right] .
$$

Then we have in view of (33)

$$
\left\|p^{n}(\mu)-p^{n}\left(\mu^{0}\right)\right\| \leq \varepsilon^{\frac{1}{2}} .
$$

If we let

$$
\eta(\varepsilon)=\varepsilon^{\frac{1}{2}}+\sum_{\max (i, j, k)>n(\varepsilon)}\left(\sum_{p, q, r=0}^{\infty} C_{i p} C_{j q} C_{k r} \mu_{p q r}^{0}\right)^{2}
$$

then, in view of (25), (26) and (34), we have $\left\|p^{n(e)}(\mu)-u_{0}\right\| \leq \eta(\varepsilon)$. As $\varepsilon \rightarrow 0$, we have $f^{-1}\left(\varepsilon^{-\frac{1}{2}}\right) \rightarrow \infty$ and $n(\varepsilon) \rightarrow \infty$. By $(8)$

$$
\sum_{\max (i, j, k)>n(e)}\left(\sum_{p, q, r=0}^{\infty} C_{i p} C_{j q} C_{k r} \mu_{p q r}^{0}\right)^{2} \rightarrow 0 \quad \text { as } n(\varepsilon) \rightarrow 0 .
$$

Hence $\eta(\varepsilon) \rightarrow 0$ as $\varepsilon \rightarrow 0$.

Now, let $u_{0} \in H^{1}(I)$. Then by Theorem 1 one has

$$
\left\|p^{n(\varepsilon)}\left(\mu^{0}\right)-u_{0}\right\| \leq \frac{\left(F\left(u_{0}\right)\right)^{\frac{1}{2}}}{n(\varepsilon)+1}
$$

The definition of $n(\varepsilon)$ implies $n(\varepsilon)+1>f^{-1}\left(\varepsilon^{-\frac{1}{2}}\right)$, and $f$ being a monotone increasing function, $f(n(\varepsilon)+1)>\varepsilon^{-\frac{1}{2}}$ or, equivalently,

$$
\frac{1}{\sqrt{8 \pi^{3}}}(3+2 \sqrt{2})^{3(n(e)+1)+3}>\varepsilon^{-\frac{1}{2}} .
$$

It follows that

$$
(3+2 \sqrt{2})^{n(\varepsilon)+2}>\sqrt{2 \pi} \varepsilon^{-\frac{1}{8}},
$$


i.e.,

$$
n(\varepsilon)+2>\frac{1}{\ln (3+2 \sqrt{2})} \ln \left(\frac{\sqrt{2 \pi}}{\varepsilon^{1 / 6}}\right) .
$$

Thus we get after some calculations

$$
n(\varepsilon)+1>\frac{\ln (\sqrt{2 \pi}(3-2 \sqrt{2}))}{\ln (3+2 \sqrt{2})}+\frac{1}{6 \ln (3+2 \sqrt{2})} \ln \left(\frac{1}{\varepsilon}\right) .
$$

It can be checked directly that the right-hand side of the latter inequality is positive if and only if

$$
0<\varepsilon<\left(\frac{\sqrt{2 \pi}}{3+2 \sqrt{2}}\right)^{6} .
$$

Let $\varepsilon$ satisfy this inequality. By the preceding arguments, (35) gives

$$
\left\|p^{n(\varepsilon)}\left(\mu^{0}\right)-u_{0}\right\| \leq \frac{\left(F\left(u_{0}\right)\right)^{\frac{1}{2}}}{C_{1}+C_{2} \ln \left(\frac{1}{\epsilon}\right)}
$$

with

$$
C_{1}=\frac{\ln (\sqrt{2 \pi}(3-2 \sqrt{2}))}{\ln (3+2 \sqrt{2})} \quad \text { and } \quad C_{2}=\frac{1}{6 \ln (3+2 \sqrt{2})}
$$

Combining (34) and (36) gives

$$
\left\|p^{n(\varepsilon)}(\mu)-u_{0}\right\| \leq \varepsilon^{\frac{1}{2}}+\frac{\left(F\left(u_{0}\right)\right)^{\frac{1}{2}}}{C_{1}+C_{2} \ln \left(\frac{1}{\varepsilon}\right)} .
$$

Now the proof of Theorem 2 will be completed once (32) is proved. In fact, one has

$$
\begin{aligned}
P_{i}(3) & =\frac{1}{\pi} \int_{0}^{\pi}(3+2 \sqrt{2} \cos \phi)^{i} d \phi \\
& =\frac{1}{\pi}\left(\int_{0}^{\pi / 2}+\int_{\pi / 2}^{\pi}\right)(3+2 \sqrt{2} \cos \phi)^{i} d \phi \\
& =\frac{1}{\pi} \int_{0}^{\pi / 2}\left((3+2 \sqrt{2} \cos \phi)^{i}+(3-2 \sqrt{2} \cos \phi)^{i}\right) d \phi .
\end{aligned}
$$

From (37), with $t=\cos \phi$, it follows that

$$
P_{i}(3) \leq \frac{2}{\pi} \int_{0}^{\pi / 2}(3+2 \sqrt{2} \cos \phi)^{i} d \phi \leq \frac{2}{\pi} \int_{0}^{1} \frac{(3+2 \sqrt{2} t)^{i}}{\sqrt{1-t^{2}}} d t \leq \frac{2}{\pi} \int_{0}^{1} \frac{(3+2 \sqrt{2} t)^{i}}{\sqrt{1-t}} d t .
$$

Putting $s=\sqrt{1-t}$ and $\zeta=s \sqrt{\frac{2 \sqrt{2}}{3+2 \sqrt{2}}}$ we get, after some computations,

$$
P_{i}(3) \leq \frac{4}{\pi}(3+2 \sqrt{2})^{i} \sqrt{\frac{3+2 \sqrt{2}}{2 \sqrt{2}}} \int_{0}^{1}\left(1-\zeta^{2}\right)^{i} d \zeta .
$$


Letting $\zeta=\cos \theta$, we have

$$
P_{i}(3) \leq \frac{4}{\pi}(3+2 \sqrt{2})^{i} \sqrt{\frac{3+2 \sqrt{2}}{2 \sqrt{2}}} \int_{0}^{\pi / 2} \sin ^{2 i+1} \theta d \theta
$$

Putting $I_{n}=\int_{0}^{\pi / 2} \sin ^{n} \theta d \theta$ we have (cf. [12: p. 681])

$$
I_{2 k}=\frac{1 \cdot 3 \cdots(2 k-1)}{2 \cdot 4 \cdots(2 k)} \cdot \frac{\pi}{2} \quad \text { and } \quad I_{2 k-1}=\frac{2 \cdot 4 \cdots(2 k-2)}{1 \cdot 3 \cdots(2 k-1)} .
$$

It follows that

$$
I_{m} I_{m-1}=\frac{\pi}{2 m} \quad(m \in \mathbb{N})
$$

But

$$
I_{m-1}=\int_{0}^{\pi / 2} \sin ^{m-1} \theta d \theta>\int_{0}^{\pi / 2} \sin ^{m} \theta d \theta=I_{m}
$$

Hence (39) gives

$$
I_{m}^{2} \leq I_{m} I_{m-1}=\frac{\pi}{2 m} .
$$

Substituting (40) into (38), we get

$$
P_{i}(3) \leq \frac{4}{\pi}(3+2 \sqrt{2})^{i} \sqrt{\frac{3+2 \sqrt{2}}{2 \sqrt{2}}} \cdot \sqrt{\frac{\pi}{2(2 i+1)}} .
$$

This establishes (32) and completes the proof of Theorem 2

Proof of Theorem 3. We choose

$$
\begin{gathered}
u_{0}(x, y, z)=\frac{1}{m(\varepsilon)} L_{m(e)+1}(x)=\frac{1}{m(\varepsilon)} L_{m(\varepsilon)+1,0,0}(x, y, z), \\
\mu_{p q r}^{0}=\int_{I} u_{0}(x, y, z) x^{p} y^{q} z^{r} d x d y d z .
\end{gathered}
$$

Since $\int_{0}^{1} L_{i}(x) x^{j} d x=0 \quad(0 \leq j<i)$ one has

$$
\mu_{p q r}^{0}=0 \quad \text { for } p \leq m(\varepsilon)
$$

i.e., $u_{0}$ is orthogonal for all the $L_{i j k}$ 's, $0 \leq i \leq m(\varepsilon)$. Put $\mu^{0}=\left(\mu_{p q r}^{0}\right)$ and $\mu=\left(\mu_{p q r}\right)$ with

$$
\mu_{p q r}=\varepsilon(-1)^{p+q+r}+\mu_{p q r}^{0} .
$$

It is clear that $\left\|\mu-\mu^{0}\right\|_{\infty}=\sup _{p, q, r}\left|\mu_{p q r}-\mu_{p q r}^{0}\right|=\varepsilon$. Hence, from assumption (15) of Theorem 3, one has .

$$
\left\|p^{m(\varepsilon)}(\mu)-u_{0}\right\| \leq \alpha(\varepsilon)\left(1+\left(F\left(u_{0}\right)\right)^{\frac{1}{2}}\right) .
$$


We estimate the left-hand side of (43). The orthonormality of the sequence $\left(L_{i j k}\right)$ implies

$$
\left\|p^{m(\varepsilon)}(\mu)-u_{0}\right\|^{2}=\left\|p^{m(\varepsilon)}(\mu)\right\|^{2}+\left\|u_{0}\right\|^{2}=\left\|p^{m(\varepsilon)}(\mu)\right\|^{2}+\frac{1}{m^{2}(\varepsilon)} .
$$

On the other hand, one has in view of (42), (29) and (31)

$$
\left\|p^{m(e)}(\mu)\right\|^{2}=\varepsilon^{2}\left(\sum_{i=0}^{m(\varepsilon)}\left(\sum_{p=0}^{i}\left|C_{i p}\right|\right)^{2}\right)^{3}=\varepsilon^{2}\left(\sum_{i=0}^{m(\varepsilon)}(2 i+1)\left|P_{i}(3)\right|^{2}\right)^{3}
$$

From (37) we conclude

$$
P_{i}(3) \geq \frac{1}{\pi} \int_{0}^{\pi / 2}(3+2 \sqrt{2})^{i} d \phi
$$

We shall make use of the inequality

$$
(2 i+1)\left|P_{i}(3)\right|^{2} \geq \frac{(3+2 \sqrt{2})^{2 i}}{2 \pi^{2}},
$$

the proof of which will be given later. Combining (47) with (45) gives

$$
\begin{aligned}
\left\|p^{m(\varepsilon)}(\mu)\right\|^{2} & \geq \varepsilon^{2}\left(\sum_{i=0}^{m(e)} \frac{(3+2 \sqrt{2})^{2 i}}{2 \pi^{2}}\right)^{3} \\
& =\frac{\varepsilon^{2}}{8 \pi^{6}}\left(\frac{(3+2 \sqrt{2})^{2(m(e)+1)}-1}{(3+2 \sqrt{2})^{2}-1}\right)^{3} \\
& \geq \frac{\varepsilon^{2}}{64 \pi^{6}}(3+2 \sqrt{2})^{6 m(\varepsilon)} .
\end{aligned}
$$

From (43), (45) and (48) we get

$$
\left(1+\left(F\left(u_{0}\right)\right)^{\frac{1}{2}}\right)^{2} \alpha^{2}(\varepsilon) \geq \frac{\varepsilon^{2}}{64 \pi^{6}}(3+2 \sqrt{2})^{6 m(\varepsilon)}+\frac{1}{m^{2}(\varepsilon)} \geq \min _{t>0} g(t),
$$

where

$$
g(t)=\frac{\varepsilon^{2}}{64 \pi^{6}}(3+2 \sqrt{2})^{6 t}+\frac{1}{t^{2}} .
$$

It is clear that $g$ attains its minimum at $t_{\varepsilon}>0$ satisfying

$$
g^{\prime}\left(t_{\varepsilon}\right)=\frac{3 \varepsilon^{2}}{32 \pi^{6}}(3+2 \sqrt{2})^{6 t} \cdot \ln (3+2 \sqrt{2})-\frac{2}{t_{\varepsilon}^{3}}=0 .
$$

From (49) one has

$$
\left(1+\left(F\left(u_{0}\right)\right)^{\frac{1}{2}}\right)^{2} \alpha^{2}(\varepsilon) \geq g\left(t_{\varepsilon}\right) \geq t_{\varepsilon}^{-2},
$$


which implies

$$
\alpha(\varepsilon) \ln \left(\frac{1}{\varepsilon}\right) \geq \frac{1}{1+\left(F\left(u_{0}\right)\right)^{\frac{1}{2}}} \cdot \frac{\ln \left(\frac{1}{\varepsilon}\right)}{t_{\varepsilon}} .
$$

From (50) one has

$$
t_{\varepsilon}^{3}(3+2 \sqrt{2})^{6 t_{\epsilon}}=\frac{64 \pi^{6}}{3 \varepsilon^{2} \ln (3+2 \sqrt{2})}
$$

Hence

$$
3 \ln t_{\epsilon}+6 t_{\epsilon} \ln (3+2 \sqrt{2})=\ln \left(\frac{64 \pi^{6}}{3 \ln (3+2 \sqrt{2})}\right)+2 \ln \left(\frac{1}{\varepsilon}\right) .
$$

Multiplying this equality by $t_{e}^{-1}$, letting $\varepsilon \rightarrow 0$ and noting that $t_{\varepsilon} \rightarrow+\infty$ as $\varepsilon \rightarrow 0$, we get

$$
\lim _{\varepsilon 10} \frac{\ln \left(\frac{1}{\varepsilon}\right)}{t_{\varepsilon}}=3 \ln (3+2 \sqrt{2}) \text {. }
$$

On the other hand, in view of (19) and the definitions of $F\left(u_{0}\right)$ and $u_{0}$ we have

$$
\begin{aligned}
F\left(u_{0}\right)= & \int_{I} x(1-x)\left|\frac{\partial u_{0}}{\partial x}(x, y, z)\right|^{2} d x d y d z \\
& +\int_{I} y(1-y)\left|\frac{\partial u_{0}}{\partial y}(x, y, z)\right|^{2} d x d y d z \\
& +\int_{I} z(1-z)\left|\frac{\partial u_{0}}{\partial z}(x, y, z)\right|^{2} d x d y d z \\
= & \frac{1}{m^{2}(\varepsilon)}(m(\varepsilon)+1)(m(\varepsilon)+2) .
\end{aligned}
$$

Hence, in view of (52) and (53), inequality (51) implies the desired inequality (16).

To complete the proof of Theorem 3 , we shall prove inequality (47). Putting $t=$ $\cos \phi$ and $s=\sqrt{1-t}$, and then letting $\zeta=s \sqrt{\frac{2 \sqrt{2}}{3+2 \sqrt{2}}}$, we get the inequality

$$
P_{i}(3) \geq \frac{1}{\pi}(3+2 \sqrt{2})^{i} \sqrt{\frac{3+2 \sqrt{2}}{\sqrt{2}}} J,
$$

where

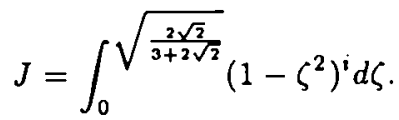

We shall estimate $J$. One has

$$
J=I_{2 i+1}-K_{i}
$$

where

$$
I_{2 i+1}=\int_{0}^{\pi / 2} \sin ^{2 i+1} \theta d \theta \quad \text { and } \quad K_{i}=\int_{\sqrt{\frac{2 \sqrt{2}}{3+2 \sqrt{2}}}}^{1}\left(1-\zeta^{2}\right)^{i} d \zeta
$$


Using (39) one has $I_{2 i+1} I_{2 i+2}=\frac{\pi}{2(2 i+2)}$. Hence

$$
I_{2 i+1}^{2} \geq I_{2 i+1} I_{2 i+2}=\frac{\pi}{2(2 i+2)}
$$

On the other hand,

$$
\begin{aligned}
K_{i} & =\int_{\frac{2 \sqrt{2}}{3+2 \sqrt{2}}}^{1}(1-\zeta)^{i} \frac{d \zeta}{2 \sqrt{\zeta}} \\
& \leq \frac{1}{2} \sqrt{\frac{3+2 \sqrt{2}}{2 \sqrt{2}}} \int_{\frac{2 \sqrt{2}}{3+2 \sqrt{2}}}^{1}(1-\zeta)^{i} d \zeta \\
& =\frac{1}{2(i+1)} \sqrt{\frac{3+2 \sqrt{2}}{2 \sqrt{2}}}\left(\frac{3}{3+2 \sqrt{2}}\right)^{i+1} .
\end{aligned}
$$

From (55) and (56) we deduce

$$
\begin{aligned}
J & \geq \sqrt{\frac{\pi}{2(2 i+2)}}-\frac{1}{2(i+1)} \sqrt{\frac{3+2 \sqrt{2}}{2 \sqrt{2}}}\left(\frac{3}{3+2 \sqrt{2}}\right)^{i+1} \\
& =\frac{1}{\sqrt{i+1}}\left(\sqrt{\frac{\pi}{4}}-\frac{1}{2 \sqrt{i+1}} \sqrt{\frac{3+2 \sqrt{2}}{2 \sqrt{2}}}\left(\frac{3}{3+2 \sqrt{2}}\right)^{i+1}\right) \\
& \geq \frac{1}{\sqrt{i+1}}\left(\sqrt{\frac{\pi}{4}}-\frac{1}{2} \sqrt{\frac{3+2 \sqrt{2}}{2 \sqrt{2}}} \cdot \frac{3}{3+2 \sqrt{2}}\right) \\
& >\frac{1}{2 \sqrt{i+1}}
\end{aligned}
$$

In view of the latter inequality, (54) gives

$$
(2 i+1)\left|P_{i}(3)\right|^{2} \geq \frac{2 i+1}{4(i+1) \pi^{2} \sqrt{2}}(3+2 \sqrt{2})^{2 i+1} \geq \frac{(3+2 \sqrt{2})^{2 i}}{2 \pi^{2}}
$$

This establishes (47) and completes the proof of Theorem 3

Acknowledgement. The work of the first author was completed with a financial support from the National Program of Basic Research of Vietnam (project "Nondestructive Evaluation"). The second author appreciates financial support from the Research Commission of Free University of Berlin (project "Convolutions") and travel grants from DFG (Deutsche Forschungsgemeinschaft) in Bonn. All three authors would like to thank the referees for their valuable comments and constructive criticisms. Our special thanks go to one of the referees for his (or her) suggestions leading to a sharpening of our estimates in Theorem 2. 


\section{References}

[1] Abramowitz, M. and I. A. Stegun: Handbook of Mathematical Functions. New York: Dover 1972.

[2] Ang, D. D., Gorenflo, R. and D. D. Trong: A multidimensional Hausdorff moment problem. Preprint. Berlin: Freie Universität, Preprint Mathematics A-23/97.

[3] Ang, D. D., Vy, L. K. and R. Gorenflo: A regularization method for the moment problem. In: Inverse Problems: Principles and Applications in Geophysics, Technology and Medicine (Math. Research: Vol. 74). Berlin: Akademie Verlag 1993, pp. $37-45$.

[4] Ang, D. D., Gorenfio, R. and L. K. Vy: Backus-Gilbert regularization of a moment problem. Preprint. Berlin: Freie Universität, Preprint Mathematics A-7/93.

[5] Askey, R., Schoenberg, I. J. and A. Sharma: Hausdorff's moment problem and expansions in Legendre polynomials. J. Math. Anal. Appl. 86 (1982), 237 - 245.

[6] Bertero, M., De Mol, C. and E. Pike: Linear inverse problems with discrete data. Part I: General formulation and singular system analysis. Inverse Problems 1 (1985), $301-330$.

[7] Inglese, G.: Recent results in the study of the moment problem. In: Theory and Practice of Geophysical Data Inversion (eds.: A. Vogel et al.). Braunschweig und Wiesbaden: Vieweg-Verlag 1992, pp. $73-84$.

[8] Kirsch, A., Schomburg, B. and G. Berendt: The Backus-Gilbert method. Inverse Problems 4 (1988), $771-783$.

[9] Lebedev, N. N.: Special Functions and Their Applications. New York: Dover Publications Inc. 1972.

[10] Rabenstein, A. L.: Introduction to Ordinary Differential Equations. New York et al.: Acad. Press 1972.

[11] Talenti, G.: Recovering a function from a finite number of moments. Inverse Problems 3 (1987), $501-517$.

[12] Taylor, A.: Advanced Calculus. New York et al.: Blaisdell Publ. Comp. 1965.

[13] Shohat, J. A. and J. D. Tamarkin: The Problem of Moments (AMS Mathematical Surveys). Providence (R.I.): Amer. Math. Soc. 1943.

Received 23.12.1997; in revised form 14.12.1998 